\title{
Adaptação e propriedades psicométricas da Escala de Atitudes Disfuncionais para Crianças e Adolescentes
}

\author{
Adaptation and psychometric properties of the Dysfunctional \\ Attitudes Scale for Children and Adolescents
}

\author{
Priscilla Moreira OHNO1 (iD) 0000-0003-0377-555X \\ Mariana Verdolin Guilherme FROESELER ${ }^{1}$ ID 0000-0002-2211-5269 \\ Maycoln Leôni Martins TEODORO ${ }^{1}$ ID 0000-0002-3021-8567
}

\begin{abstract}
Resumo
Esta pesquisa é constituída por três estudos que investigaram as propriedades psicométricas da adaptação para o português da Escala de Atitudes Disfuncionais para Crianças, instrumento de autorrelato que avalia componentes da vulnerabilidade cognitiva para a depressão em jovens. Crianças e Adolescentes ( $n=596 ; 9$ a 16 anos) completaram a escala e outros instrumentos de avaliação de cognições e sintomas depressivos. Foram encontrados bons índices de consistência interna para o instrumento, e correlações moderadas a fracas entre a escala no teste-reteste ( $n=225$; intervalo médio de 8,3 meses) e os demais instrumentos. Análises fatoriais exploratórias $(n=218)$ e confirmatórias ( $n=378$ ) evidenciaram a estrutura trifatorial. Os resultados apontam a Escala de Atitudes Disfuncionais para Crianças como uma medida adequada de atitudes disfuncionais em jovens.
\end{abstract}

Palavras-chave: Adolescentes; Atitudes disfuncionais; Cognição; Crianças; Depressão; Escala.

\begin{abstract}
Three studies investigated the psychometric properties of the adaptation to Portuguese of the Dysfunctional Attitude Scale for Children, a self-report instrument that assesses components of cognitive vulnerability to depression in young people. Children and adolescents ( $n=596 ; 9$ to 16 years) completed the scale and other cognitions assessment tools and depressive symptoms. There were found good internal consistency indices for the instrument, and moderate to week correlations between the Disfunctional Attitude Scale of Children in the test-retest reliability $(n=225$; mean interval of 8.3 months) and with the other instruments. Exploratory $(n=218)$ and confirmatory $(n=378)$ factor analysis showed a three factor structure. The results indicate that Dysfunctional Attitude Scale for Children is a suitable measurement of dysfunctional attitudes in young people.
\end{abstract}

Keywords: Adolescents; Dysfunctional attitude; Cognition; Children; Depression; Scale.

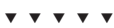

1 Universidade Federal de Minas Gerais, Faculdade de Filosofia e Ciências Humanas, Programa de Pós-Graduação em Psicologia: Cognição e Comportamento. Av. Presidente Antônio Carlos, 6627, Pampulha, 31270-901, Belo Horizonte, MG, Brasil. Correspondência para/ Correspondence to: M.L.M. TEODORO.E-mail: <mlmteodoro@hotmail.com>

Apoio: Fundação de Amparo à Pesquisa do Estado de Minas Gerais e Conselho Nacional de Desenvolvimento Científico e Tecnológico.

$\boldsymbol{\nabla} \boldsymbol{\nabla} \boldsymbol{V}$

Como citar este artigo/How to cite this article

Ohno, P. M., Froeseler, M. V. G., \& Teodoro, M. L. M. (2018). Adaptação e propriedades psicométricas da Escala de Atitudes Disfuncionais para Crianças e Adolescentes. Estudos de Psicologia (Campinas), 35(2), 171-180. http://dx.doi.org/10.1590/1982-02752018000200006
} 
O modelo cognitivo desenvolvido por Aaron T. Beck destaca a importância dos esquemas cognitivos depressogênicos que orientam a visão e interpretação que o indivíduo faz dos eventos que vivencia (Beck \& Alford, 2011). Esquemas são padrões cognitivos idiossincráticos e estáveis que funcionam abaixo do nível da consciência e que atuam como filtros das informações e experiências recebidas pelo indivíduo, organizando, classificando e definindo a leitura que ele fará da realidade. São constituídos pelas crenças centrais, ideias mais profundas e rígidas que o indivíduo desenvolve ao longo de sua infância e adolescência, que vão se fortalecendo e se consolidando em um estilo de pensamento específico próprio. Associadas às crenças centrais estão as crenças condicionais subjacentes, compostas por pressupostos e regras que orientam o indivíduo sobre o modo como deve agir a fim de que possa suportar e manter, mesmo que inadequadamente, suas crenças centrais.

O desenvolvimento e a consolidação das crenças ocorrem na infância e adolescência, e estão relacionados com a personalidade e o desenvolvimento emocional e cognitivo do indivíduo, sofrendo, ainda, influência de seu ambiente interpessoal, e dependendo de habilidades cognitivas específicas, como metacognição e memória (Teodoro \& Ohno, 2017). Os esquemas mal adaptativos, ou disfuncionais, levam os indivíduos a fazerem avaliações distorcidas de sua realidade (Beck \& Alford, 2011).

Cada indivíduo possui características internas e estáveis que o predispõem a realizar construções cognitivas falhas específicas (distorções cognitivas), podendo conduzi-lo ao desenvolvimento de um transtorno (Ingram, 2003). Na depressão, essa vulnerabilidade cognitiva é proveniente da tríade cognitiva negativa, que corresponde ao conjunto de pensamentos e atitudes negativas duradouras que o indivíduo tem sobre si mesmo, o mundo e seu futuro (Beck \& Alford, 2011). Se, durante a infância, o indivíduo desenvolve crenças disfuncionais, ele pode se tornar vulnerável à depressão, podendo ativar, futuramente, tais crenças diante de um evento estressor (Ingram, 2003). A ativação dessas crenças latentes gera um padrão de processamento de informação de autorreferência negativo, que conduz à depressão. Esse processamento falho de informações faz com que a pessoa deprimida tenda a estruturar o seu pensamento de maneira negativa, fazendo julgamentos amplos, extremos e categóricos com relação à sua vida, mesmo diante de evidências contraditórias (Beck \& Alford, 2011).

Para avaliar o processamento cognitivo característico da depressão, Weissman e Beck (1978) desenvolveram a Dysfunctional Attitude Scale (DAS, Escala de Atitudes Disfuncionais), instrumento que seria capaz de identificar as cognições subjacentes que constituem fator de vulnerabilidade para essa patologia em adultos. A versão final dessa escala de autorrelato, baseada na teoria de Beck, contém duas formas curtas paralelas (DAS-A e DAS-B) com quarenta itens cada, sendo a DAS-A a versão mais utilizada com confiabilidade teste-reteste de 0,84 . A DAS foi concebida inicialmente como uma medida unidimensional; no entanto, outros estudos apresentaram dois (Graaf, Roelofs, \& Huibers, 2009), três (Power et al., 1994) e quatro fatores (Oliver \& Baumgart, 1985), não havendo assim um consenso na literatura. O instrumento foi adaptado para o Brasil por Orsini, Tavares e Tróccoli (2006), apresentando uma estrutura trifatorial (baixa autoestima indutora, baixa autoestima induzida por objeto esquemático e atitudes mantenedoras da depressão), com índices de consistência interna que variaram de 0,57 a 0,71 para a versão $A$, e de 0,64 a 0,77 para a versão $B$.

A interação entre atitudes disfuncionais e eventos estressores conduz pessoas adultas a um quadro depressivo (Otto et al., 2007). No público mais jovem, sabe-se que adolescentes com altos níveis de atitudes disfuncionais experienciam mais sintomas depressivos, principalmente aqueles que passaram por eventos tidos como estressores (Young, LaMontagne, Dietrich, \& Wells, 2012). A presença de sintomas depressivos em crianças é relativamente semelhante entre meninos e meninas na infância. No entanto, por volta dos 15 anos de idade, essa diferença começa a se acentuar, tendo as meninas uma probabilidade duas vezes maior do que os meninos de desenvolverem um quadro depressivo (Villano \& Nanhay, 2011). 
Especificamente em relação a atitudes disfuncionais, verificam-se resultados discrepantes quanto a diferenças de gênero. Marcotte, Marcotte e Boufard (2002) encontraram maiores índices de atitudes disfuncionais relacionadas a sucesso e autocontrole entre adolescentes canadenses do sexo masculino. Em contraponto, Rogers et al. (2013) avaliaram atitudes disfuncionais em 439 adolescentes com Transtorno Depressivo Maior e não encontraram diferenças significativas entre meninos e meninas.

Destinada à avaliação sistemática de atitudes disfuncionais em crianças e adolescentes, a Dysfunctional Attitudes Scale for Children (DAS-C, Escala de Atitudes Disfuncionais para Crianças e Adolescentes) foi desenvolvida por D'Alessandro e Burton (2006), tendo como base inicial a versão adulta da escala. O processo de adaptação contou com modificações na linguagem e exclusão de itens confusos e repetitivos, que foram seguidos de análises fatoriais e de correlação item-total que contribuíram para a seleção dos 22 itens que compõem a escala. No estudo original, a DAS-C apresentou estrutura unifatorial e índices adequados de confiabilidade, com um alfa de Cronbach de 0,87 e confiabilidade teste-reteste de 0,80. Também foram encontradas correlações positivas moderadas entre a DAS-C e as medidas de depressão, afeto negativo, visão negativa da tríade cognitiva, autocrítica e neuroticismo.

Diante do exposto, observa-se que a DAS-C é um instrumento com boas propriedades psicométricas para a avaliação de atitudes disfuncionais em crianças e adolescentes. Considerando a ausência de uma versão desse instrumento no contexto brasileiro e a importância de avaliar esse construto tanto na prática clínica quanto na pesquisa científica, este estudo objetiva adaptar e investigar algumas propriedades psicométricas da DAS-C. Para tal, foram conduzidos três estudos. No primeiro, realizou-se a tradução, adaptação e verificação da estrutura fatorial do instrumento Análise Fatorial Exploratória (AFE); no segundo, foi feita uma análise fatorial confirmatória; por fim, um terceiro estudo foi conduzido a fim de verificar as evidências de validade convergente e estabilidade interna do instrumento, além de diferenças em relação a sexo.

\section{Estudo 1}

Esse estudo transversal teve como principal objetivo realizar a tradução e adaptação da Dysfunctional Attitudes Scale for Children para o português. Também foram verificadas algumas de suas propriedades psicométricas preliminares (estrutura fatorial e confiabilidade).

\section{Método}

\section{Participantes}

Trata-se de uma amostra selecionada por conveniência, composta por 218 crianças e adolescentes de nove a 16 anos (Média = 12,28; $D P=1,59$ ), alunos de escolas públicas e particulares de Belo Horizonte e região metropolitana. A amostra foi constituída por $55,8 \%$ de participantes do sexo feminino $(n=121)$.

\section{Instrumento}

A Escala de Atitudes Disfuncionais para Crianças e Adolescentes é a versão adaptada da Dysfunctional Attitudes Scale for Children (DAS-C), (D'Alessandro \& Burton, 2006). Trata-se de uma medida desenvolvida para investigar as atitudes disfuncionais em crianças e adolescentes composta por 22 itens, respondidos em uma escala Likert de seis pontos, variando de discordo fortemente até concordo fortemente.

\section{Procedimentos}

A Dysfunctional Attitudes Scale for Children passou por um processo de tradução para o português, no qual três juízes produziram suas próprias versões de forma independente. Posteriormente, as versões foram comparadas e os itens discutidos um a um por dois juízes com experiência na língua inglesa e psicologia, chegando-se assim a uma versão única da escala, que foi retraduzida para o 
inglês. Essa versão em inglês foi comparada com a versão original por uma profissional bilíngue, resultando na versão final da escala.

A aplicação dos instrumentos em ambos os estudos foi coletiva, realizada em sala de aula, após o convite ter sido feito aos alunos. Estes entregaram o Termo de Consentimento Livre Esclarecido assinado por seus responsáveis. A aplicação durou, em média, 50 minutos, durante os quais se pediu para que o professor se retirasse da sala para evitar eventuais constrangimentos para os alunos. O projeto de pesquisa foi aprovado pelo Comitê de Ética da Universidade Vale dos Sinos (Unisinos) (Protocolo n0041.0.390.000-09).

\section{Análise dos dados}

A estrutura fatorial da DAS-C foi avaliada por meio de AFE, após a verificação da normalidade da distribuição dos dados por meio do teste de Kolmogorov-Smirnov. Todas as variáveis analisadas não apresentaram distribuição normal; no entanto, optou-se pelo uso de testes paramétricos uma vez que o tamanho amostral justifica tal utilização. A fim de verificar a possibilidade de fatoração dos dados, foram analisados dois índices: a medida de adequação da amostra de Kaiser-Meyer-Olkin (KMO) e o teste de esfericidade de Bartlett. A escolha dos fatores a serem extraídos foi feita a partir da hipótese teórica, do percentual de variância explicada, do critério de Kaiser e da análise do gráfico de sedimentação. Evidências da fidedignidade do instrumento foram obtidas por meio de análise da consistência interna da escala e análise dos itens (cargas fatoriais e coeficientes de correlação item-total).

\section{Resultados e Discussão}

$\mathrm{Na}$ AFE, conduzida pelo método de extração Principais Eixos Fatoriais (PAF) e rotação Varimax, o Teste de Bartlett $(p<0,01)$ e o $\operatorname{KMO}(0,85)$ indicaram a possibilidade de fatoração dos dados. A partir do critério de Kaiser foram identificados seis fatores, que explicam 57,02\% da variância total, sendo que o primeiro fator apresentou autovalor
$(6,22)$ e percentual de variância explicada $(28,26 \%)$ consideravelmente discrepantes dos demais fatores. A análise do gráfico de sedimentação indica uma estabilização após o terceiro fator, sugerindo uma estrutura trifatorial.

A partir dos resultados da AFE inicial foram conduzidas novas AFE solicitando a extração de um, três e seis fatores. A solução de seis fatores não se mostrou adequada, uma vez que muitos itens carregaram concomitantemente em mais de um fator, e com cargas fatoriais próximas umas das outras (o item 1, por exemplo, carregou em quatro fatores com a carga fatorial variando de 0,30 a 0,46). Além disso, a distribuição dos itens não se deu de forma homogênea, tendo fatores com apenas dois ou três itens. A solução trifatorial explicou $42,08 \%$ da variância total e os fatores se correlacionaram de forma significativa, porém as magnitudes dessas correlações foram baixas ( 1 e 2 : $r=0,56 ; 1$ e $3: r=0,50 ; 2$ e $3: r=0,51$ ).

Considerando que as análises do gráfico de sedimentação e do critério de Kaiser, além da perspectiva teórica, apontaram para diferentes quantidades de fatores a serem retidos, decidiu-se por conduzir uma análise paralela (Damásio, 2012). Essa nova análise sugeriu um modelo de quatro fatores $\left(\chi^{2}=230,58 ; p<0,001\right.$; RMSEA $\left.=0,05\right)$, no qual quatro itens tiveram cargas fatoriais abaixo de 0,30. Além disso, cinco itens apresentaram cargas fatoriais superiores a 0,3 em mais de um fator. Cabe destacar que a organização dos itens entre os quatro fatores não apresentou suficiente consistência teórica.

Todos os coeficientes de correlação item-escala total apresentaram-se acima de 0,30, com exceção do item 21 "Eu sempre devo ser capaz de resolver as coisas sozinho (a)", que teve uma correlação de 0,25 no modelo unifatorial. Nesse mesmo modelo, os demais valores variaram de 0,33 (Item 1 "As pessoas só vão gostar de mim se eu for bom sempre") a 0,62 (Item 20 "Você tem que impressionar as outras crianças para que elas gostem de você") no modelo com uma única variável latente. Já no modelo trifatorial, os valores foram de 0,31 (Item 21 na subescala Aprovação social) a 0,63 (Item 14 na subescala Autodesempenho e Amor). 
Quanto às cargas fatoriais, somente o item 21, no modelo unifatorial, apresentou o valor de 0,28 ; todos os demais itens (tanto no modelo unifatorial quanto no trifatorial) apresentaram valores superiores a 0,30, que, segundo Laros (2012), corresponde ao valor mínimo para que o item seja um representante útil do fator. A análise das cargas fatoriais apresentou valores adequados, mostrando que os itens podem ser tidos como bons representantes do traço latente em questão.

\section{Estudo 2}

Esse estudo teve como objetivo testar a estrutura fatorial da DAS-C por meio de Análises Fatoriais Confirmatórias (AFC).

\section{Método}

\section{Participantes}

Participaram desse estudo 378 crianças e adolescentes de 10 a 16 anos (Média = 12,48; $D P=1,25)$, alunos de escolas públicas e particulares de Belo Horizonte e região metropolitana. Os participantes do sexo feminino eram 220, correspondentes a 58,2\% da amostra.

Os instrumentos e procedimentos de coleta de dados do Estudo 2 foram iguais aos relatados no Estudo 1.

\section{Análise dos dados}

A Análise Fatorial Confirmatória (AFC) da DAS-C foi realizadacom o método de estimação Maximum Likelihood (ML). A adequação do modelo foi avaliada, no estudo, segundo alguns indicadores. O primeiro consiste na razão entre o qui-quadrado e os graus de liberdade $\left(\chi^{2} / g L\right)$, sendo o ajuste do modelo indicado por valores inferiores a cinco. Também foram avaliados o Comparative Fit Index (CFI) e o Root Mean Square Error of Approximation (RMSEA). O primeiro deve apresentar valor superior a 0,90, enquanto o segundo deve ser inferior a 0,10 (valores menores que 0,05 indicam ótimo ajuste do modelo).
Diante dos resultados das AFE do Estudo 1, nos quais o gráfico de sedimentação e a análise do agrupamento dos itens apontaram divergências, optou-se pelo cálculo e comparação dos modelos com um e três fatores. Optou-se pela exclusão dos modelos de quatro e de seis fatores, considerando indicadores estatísticos (baixa variância explicada, itens com cargas fatoriais abaixo de 0,30 e poucos itens nos últimos fatores) e teóricos.

\section{Resultados e Discussão}

O resumo das análises da estrutura fatorial da DAS-C encontra-se descrito na Tabela 1. O modelo unifatorial que representa a estrutura original do instrumento (D'Alessandro \& Burton, 2006) foi calculado de modo que os 22 itens da DAS-C fossem explicados por uma única variável latente. O modelo trifatorial foi elaborado com base nas AFE realizadas no Estudo 1. Ambos os modelos apresentaram índices de ajuste apropriados e encontram-se dentro dos critérios de interpretação utilizados como referência. No entanto, o modelo trifatorial foi o que apresentou os melhores índices: valores menores do Akaike Information Criterion (AIC) $(616,31)$, do quociente $\chi^{2} / g L(3,14)$ e RMSEA $(0,06)$; e maiores CFI $(0,95)$ e Non-Normed Fit Index (NNFI)(0,95). Sendo assim, todos os índices avaliados indicaram a melhor adequação do modelo trifatorial.

A partir da análise dos itens e seu respectivo agrupamento, verificou-se que o fator 1 representa regras e suposições condicionais acerca do perfeccionismo e avaliação crítica e negativa relacionada a ser amado ou ao seu próprio desempenho, sendo, portanto, chamado de Autodesempenho e Amor. Já o segundo fator representa regras e metas que a pessoa impõe para si mesma e sua preocupação com críticas e expectativas dos outros, tendo sido nomeado de Autocobrança e Necessidade de aprovação. Por fim, o terceiro fator reflete regras e atitudes rígidas relativas a características que são socialmente positivas, sendo assim denominado de Aprovação social.

As cargas fatoriais dos itens da DAS-C variaram de 0,31 a 0,68 (Média $=0,53 ; D P=0,09$ ) na solução unifatorial e de 0,35 a 0,71 (Média = 0,57; $D P=0,08)$ no modelo trifatorial. As correlações 
Tabela 1

Resumo dos resultados para os modelos de um e três fatores da Escala de Atitudes Disfuncionais para Crianças e Adolescentes

\begin{tabular}{lccccccc}
\hline Modelo & $\chi^{2}$ & GL & $\chi^{2} / G L$ & RMSEA & CFI & NNFI & AIC \\
\hline 1 Fator & 656,19 & 209 & 3,139 & 0,083 & 0,94 & 0,93 & 841,01 \\
3 Fatores & 522,26 & 206 & 2,535 & 0,064 & 0,95 & 0,95 & 616,31 \\
\hline
\end{tabular}

Nota: $\chi^{2}$ : Valor do Qui-Quadrado; GL: Grau de Liberdade; RMSEA: Root Mean Squared of the Residuals; CFI: Comparative Fit Index; NNFI: The Tucker-Lewis Index (Non-Normed Fit Index); AIC: Akaike Information Criterion.

dos itens com o total de cada fator mostraram três escores inferiores a 0,40 , sendo que dois deles se encontram no fator 2 (itens 4 e 21) e um no fator 3 (item 10). Verificou-se nesse estudo que os Alfas de Cronbach foram de 0,71 (Aprovação social), 0,82 (Autodesempenho e Amor) e 0,76 (Autocobrança e Necessidade de aprovação); sendo de 0,89 para a escala total, indicando assim um índice de precisão do instrumento próximo a excelente (Hauck Filho \& Zanon, 2015).

\section{Estudo 3}

Esse estudo foi conduzido a fim de verificar a estabilidade temporal e a validade convergente da DAS-C. Além disso, buscou-se analisar a possível associação das atitudes disfuncionais com sintomas depressivos na população estudada.

\section{Método}

\section{Participantes}

Os participantes dos Estudos 1 e 2 foram agrupados, compondo assim uma amostra de 596 crianças e adolescentes, com idade entre nove e 16 anos (Média $=12,41 ; D P=1,39$ ), sendo $57,3 \%$ de meninas ( $n=341$ ). Desse total, 225 responderam aos mesmos instrumentos após um intervalo médio de 8,3 meses da primeira aplicação. Nessa subamostra, a idade dos participantes variou entre 10 e 16 anos (Média $=12,39 ; D P=1,17$ ), sendo $56,9 \%$ de meninas $(n=128)$.

\section{Instrumentos}

Foram utilizados nesse estudo os quatro instrumentos abaixo descritos.
1) Escala de Atitudes Disfuncionais para Crianças e Adolescentes (DAS-C): (D'Alessandro \& Burton, 2006), descrita no Estudo 1.

2) Inventário da Tríade Cognitiva para Crianças e Adolescentes (ITC-CA): O ITC-CA é composto por 36 itens que avaliam as visões de self, mundo e futuro de crianças e adolescentes, em uma escala Likert de três pontos (sim, talvez e não). A adaptação brasileira realizada por Teodoro, Froeseler, Almeida e Ohno (2015) apresentou uma estrutura com seis componentes (Self Positivo; Self Negativo; Mundo Positivo; Mundo Negativo; Futuro Positivo; Futuro Negativo) e coeficientes de consistência interna adequados variando de 0,61 a 0,74 nas subescalas.

3) Escala de Pensamentos Automáticos para Crianças e Adolescentes (EPA): A EPA possui 40 itens que investigam pensamentos automáticos em crianças e adolescentes, distribuídos em quatro fatores (fracasso pessoal, ameaça social, ameaça física e hostilidade), por meio de uma escala Likert de cinco pontos (variando de nunca até todo o tempo). A adaptação para o português foi realizada por Teodoro, Andrade e Castro (2013), que encontraram índices de consistência interna variando de 0,76 a 0,91 para os fatores.

4) Inventário de Depressão Infantil (CDI): O CDI possui 27 itens que investigam a intensidade dos sintomas de depressão em crianças e adolescentes. Os participantes precisam escolher a alternativa que melhor descreve o seu estado emocional na atualidade, por meio de uma escala Likert de três pontos. A adaptação do CDI para o Brasil foi feita por Gouveia, Barbosa, Almeida e Gaião (1995), apresentando consistência interna de 0,81. Considerando a subamostra que preencheu o CDI 
nesse estudo ( $n=434)$, foi encontrado um Alfa de Cronbach de 0,85 para essa escala.

\section{Procedimentos}

A coleta dos dados foi coletiva, realizada em sala de aula e com duração de aproximadamente 50 minutos. Os participantes dos Estudos 1 e 2 foram recontatados após oito meses e convidados a preencher os instrumentos anteriormente descritos. Foram seguidos os procedimentos éticos dos dois estudos anteriores.

\section{Análise dos Dados}

A fidedignidade da DAS-C foi avaliada por meio da estabilidade teste-reteste, com intervalo de 8,3 meses entre as aplicações. Utilizou-se, também, o Teste $t$ para amostras pareadas nessa verificação da estabilidade, considerando-se dois grupos etários distintos. Foram empreendidas análises de correlação entre a DAS-C e os demais instrumentos, considerando que tríade cognitiva, atitudes disfuncionais, pensamentos automáticos e sintomas depressivos são construtos relacionados no modelo cognitivo da depressão. Também foi conduzida análise de comparação entre meninos e meninas, por meio do Teste $t$ de Student para amostras independentes.

\section{Resultados e Discussão}

Em relação à análise da fidedignidade da DAS-C, via teste-reteste, foram encontradas correlações de 0,42 (Aprovação social), 0,43 (Autodesempenho e Amor), 0,51 (Autocobrança e Necessidade de aprovação) e de 0,50 para escala total entre a primeira e a segunda avaliação. $\mathrm{O}$ coeficiente de correlação encontrado nessa análise $(r=0,50)$ foi significativo, porém inferior ao do instrumento original $(r=0,80)$. Tal fato pode ser devido à discrepância de tempo entre as avaliações; no presente estudo o intervalo médio foi de 8,3 meses, enquanto no estudo original esse intervalo foi de apenas três semanas. Apesar de inferior à encontrada por D'Alessandro e Burton (2006), a correlação encontrada nesse estudo é moderada (Dancey \& Reidy, 2011), indicando uma estabilidade nas atitudes disfuncionais. Por se tratar de estudo conduzido com amostra de idade variável (10 a 16 anos; Média $=12,39 ; D P=1,17$ ), é possível hipotetizar que tal estabilidade esteja sob influência da idade, sendo maior entre os participantes mais velhos. Para testar essa hipótese, a amostra foi dividida em dois grupos etários: (1) de 10 a 12 anos $(n=123)$ e (2) de 13 a 16 anos $(n=102)$. O coeficiente de correlação que indica a estabilidade das atitudes disfuncionais foi maior no grupo 2 em todos os fatores, com exceção do fator Autocobrança e Necessidade de aprovação, que foi menor (Tabela 2). Análises de frequência indicaram que houve uma diminuição do escore médio dos participantes na segunda etapa da avaliação em comparação à primeira. Para verificar se essa diferença entre as médias foi significativa, utilizou-se o Teste $t$ para amostras pareadas. Os resultados indicaram que a diferença é significativa apenas para o grupo 1 na subescala Autodesempenho e Amor $(p<0,01)$, sendo que no tempo 1 a média foi $16,03(D P=7,03)$, no tempo 2 foi $14,14(D P=5,23)$ e na escala total $(p<0,05$; T1: Média $=51,72$; $D P=18,11$ e T2: Média $=48,09 ; D P=15,39$ ).

Sendo assim, os resultados encontrados indicam que diferentes tipos de atitudes disfuncionais se estabilizam em diferentes momentos da infância e adolescência. Hipotetiza-se que a diminuição da disfuncionalidade dessas crenças pode estar relacionada com o desenvolvimento de habilidades metacognitivas (pensar sobre o próprio pensamento), que pode fazer com que a criança esteja mais consciente e seja mais crítica nesse processo de consolidação das crenças.

Todas as análises de correlação entre a DAS-C e os fatores da EPA e ITC-CA resultaram em coeficientes estatisticamente significativos, mas de baixa magnitude. As correlações variaram de -0,13 (Futuro Positivo; ITC-CA e Autocobrança e Necessidade de aprovação; DAS-C) a 0,48 (Self Negativo; ITC-CA e Autodesempenho e Amor; DAS-C) (Tabela 2). 
Correlações entre a Escala de Atitudes Disfuncionais para Crianças e Adolescentes (DAS-C) e os fatores da versão brasileira do ITC-CA e a Escala de Pensamentos Automáticos (EPA)

\begin{tabular}{|c|c|c|c|}
\hline & \multicolumn{3}{|c|}{ DAS-C } \\
\hline & DesAm & Cob & Aprovação \\
\hline \multicolumn{4}{|l|}{ ITC-CA } \\
\hline SP & $-0,36^{* *}$ & $-0,15^{* *}$ & $-0,18^{* *}$ \\
\hline SN & $0,48^{* *}$ & $0,32^{* *}$ & $0,34^{* *}$ \\
\hline MP & $-0,28^{* *}$ & $-0,15^{* *}$ & $-0,30^{* *}$ \\
\hline MN & $0,41^{* *}$ & $0,31^{* *}$ & $0,33^{* *}$ \\
\hline FP & $-0,33^{* *}$ & $-0,13^{* *}$ & $-0,19^{* *}$ \\
\hline FN & $0,46^{* *}$ & $0,29^{* *}$ & $0,32^{* *}$ \\
\hline \multicolumn{4}{|l|}{ EPA } \\
\hline $\mathrm{FPe}$ & $0,48^{* *}$ & $0,35^{* *}$ & $0,32^{* *}$ \\
\hline AS & $0,43^{* *}$ & $0,35^{* *}$ & $0,31^{\star *}$ \\
\hline$A F$ & $0,40^{* *}$ & $0,26^{* *}$ & $0,27^{\star \star}$ \\
\hline $\mathrm{HO}$ & $0,30^{* *}$ & $0,28^{* *}$ & $0,30^{* *}$ \\
\hline \multicolumn{4}{|l|}{$C D$} \\
\hline D & $0,41^{* *}$ & $0,21^{* *}$ & $0,28^{* \star}$ \\
\hline \multicolumn{4}{|c|}{ DAS-C (teste-reteste) } \\
\hline Grupo 1 & $0,29^{* *}$ & $0,56^{* *}$ & $0,37^{* *}$ \\
\hline Grupo 2 & $0,54^{* *}$ & $0,43^{* *}$ & $0,49^{* *}$ \\
\hline
\end{tabular}

Nota: ${ }^{* *} p<0,01$; DesAm: Autodesempenho e Amor; Cob: Autocobrança e Necessidade de Aprovação; Aprov: Aprovação Social; SP: Self Positivo; SN: Self Negativo; MP: Mundo Positivo; MN: Mundo Negativo; FP: Futuro Positivo; FN: Futuro Negativo; FPe: Fracasso Pessoal; AS: Ameaça Social; AF: Ameaça Física; HO: Hostilidade; D: Depressão; Grupo 1: participantes com idade entre 10 e 12 anos; Grupo 2: Participantes com idade entre 13 e 16 anos.

As correlações moderadas a fracas entre os escores da DAS-C e os demais instrumentos indicam que as atitudes disfuncionais se relacionam, mas não se sobrepõem a outros construtos relacionados à depressão (tríade cognitiva negativa e pensamentos automáticos disfuncionais). Também foram encontradas associações significativas $(p<0,01)$ entre os fatores da DAS-C e o CDI, que variaram de 0,21 (Autocobrança e Necessidade de aprovação) a 0,41 (Autodesempenho e Amor). Resultados semelhantes entre baixas correlações de atitudes disfuncionais e sintomas depressivos foram encontrados na versão brasileira da escala para adultos (Orsini et al., 2006).

A baixa variância comum entre os fatores da DAS-C e o CDI pode ser atribuída ao fato de que esse estudo foi conduzido com uma amostra não clínica, o que levaria à baixa representatividade das atitudes disfuncionais comumente encontradas em crianças e adolescentes deprimidos. Outra possível explicação para tais resultados é que a DAS-C possa não ser um instrumento específico, utilizado somente para a avaliação da vulnerabilidade cognitiva para a depressão. Apesar de muitas pessoas apresentarem esquemas depressogênicos relacionados com autoavaliações e relações interpessoais negativas, nem todas irão desenvolver um quadro depressivo. No entanto, esses esquemas negativos podem estar relacionados ao desenvolvimento e manutenção de outros problemas e/ou transtornos psicológicos. Sendo assim, recomenda-se que estudos futuros levem em consideração a avaliação de possíveis correlações da DAS-C com outras medidas de sintomas além dos depressivos.

As análises de comparação entre grupos, em relação ao sexo dos participantes, mostraram que apenas no que diz respeito a Aprovação social os meninos (Média $=12,33 ; D P=5,93$ ) apresentam 
mais atitudes disfuncionais do que as meninas (Média $=11,05 ; D P=5,20)(t=2,75 ; p<0,01)$, mas a diferença apresenta pequeno tamanho de efeito $(d=0,23)$. Esse resultado encontra suporte parcial nos achados de Marcotte et al. (2002), que verificaram maiores níveis de atitudes disfuncionais em adolescentes do sexo masculino. A aproximação entre os resultados encontra-se limitada devido ao tamanho do efeito encontrado no presente estudo e a diferenças nos instrumentos utilizados (DAS-C versus forma alternativa da DAS). Não foram encontradas diferenças estatisticamente significativas nos outros dois fatores.

\section{Considerações Finais}

Identificar as atitudes disfuncionais é um importante passo no processo psicoterápico, uma vez que é a partir do conhecimento de quais são as estratégias cognitivas que geralmente as pessoas utilizam para manterem suas crenças centrais disfuncionais que se consegue modificar os padrões desadaptativos que Ihes ocasionam algum grau de sofrimento. A DAS-C apresentou propriedades psicométricas adequadas, demonstrando assim ser um bom instrumento para identificar as atitudes disfuncionais presentes no público mais jovem. Ao se analisar a estabilidade dessas atitudes, percebeu-se que com o passar do tempo elas tendem a diminuir. Tal resultado deve ser mais bem investigado em estudos futuros que utilizem a metodologia da psicologia do desenvolvimento, em delineamentos sequenciais ou mesmo de coorte, para se testarem as hipóteses levantadas no presente estudo, que não utilizou tais recursos metodológicos.

Sendo assim, a DAS-C se mostra um instrumento que pode auxiliar na detecção e verificação de mudanças nos esquemas depressogênicos de crianças e adolescentes. Além disso, sendo a DAS-C um instrumento utilizado como medida de vulnerabilidade cognitiva para depressão, o seu uso pode, inclusive, auxiliar em trabalhos preventivos de futuros quadros depressivos nesse público. Nesse sentido, a tradução e adaptação dessa escala irá suprir uma lacuna existente no contexto nacional para tal investigação. No entanto, os resultados aqui encontrados devem ser interpretados com cautela, uma vez que a amostra não foi selecionada aleatoriamente, e não possui representantes de grupos clínicos ou de outras cidades brasileiras.

\section{Colaboradores}

P.M. OHNO e M.V.G. FROESELER: desenho e redação do artigo, coleta, análise e interpretação dos dados; ambas são doutorandas em Psicologia. M.L.M. TEODORO: concepção, revisão e aprovação da versão final do artigo.

\section{Referências}

Beck, A. T., \& Alford, B. A. (2011). Depressão: causas e tratamento (2a. ed.). Porto Alegre: Artmed.

D'Alessandro, D. U., \& Burton, K. B. (2006). Development and validation of the Dysfunctional Attitudes Scale for Children: Tests of Beck's cognitive diathesisstress theory of depression, of its causal mediation component, and of developmental effects. Cognition Therapy Research, 30(3), 335-353. http://dx.doi. org/10.1007/s10608-006-9046-5

Damásio, B. F. (2012). Uso da análise fatorial exploratória em psicologia. Avaliação Psicológica, 11(2), 213-228. Recuperado em abril 19, 2018, de http://pepsic. bvsalud.org/pdf/avp/v11n2/v11n2a07.pdf

Dancey, C. P., \& Reidy, J. (2011). Statistical without math for psychology (5th ed). Harlow: Pearson Education.

Gouveia, V., Barbosa, G., Almeida, H., \& Gaião, A. (1995). Inventário de Depressão Infantil: CDI: estudo de adaptação com escolares de João Pessoa. Jornal Brasileiro de Psiquiatria, 44(7), 345-349.

Graaf, L. E., Roelofs, J., \& Huibers, M. J. H. (2009). Measuring Dysfunctional Attitudes in the general population: The Dysfunctional Attitude Scale (form A) Revised. Cognitive Therapy and Research, 33(4), 345-355. http://dx.doi.org/10.1007/s10608-009-9229-y

Hauck Filho, N., \& Zanon, C. (2015). Questões básicas sobre mensuração. In C. S. Hutz, D. R. Bandeira, \& C. Trentini (Orgs.), Psicometria. Porto Alegre: Artmed.

Ingram, R. E. (2003). Origins of cognitive vulnerability to depression. Cognitive Therapy and Research, 27(1), 77-88. http://dx.doi.org/0147-5916/03/0200-0077/0

Laros, J. A. (2012). O uso da análise fatorial: algumas diretrizes para pesquisadores. In L. Pasquali (Ed.), Análise fatorial para pesquisadores (pp.141-160). Brasília: LabPAM Saber e Tecnologia.

Marcotte, G., Marcotte, D., \& Bouffard, T. (2002). The influence of familial support and dysfunctional attitudes 
on depression and delinquency in an adolescent population. European Journal of Psychology of Education, 17(4), 363-376. http://dx.doi.org/10.1007/ BF03173591

Oliver, J. M., \& Baumgart, E. P. (1985). The Dysfunctional Attitude Scale: Psychometric properties and relation to depression in an unselected adult population. Cognitive Therapy and Research, 9(2), 161-167. http:// dx.doi.org/10.1007/BF01204847

Orsini, M. R. C. A., Tavares, M., \& Tróccoli, B. T. (2006). Adaptação brasileira da Escala de Atitudes Disfuncionais (DAS). Psico-USF, 11(1), 25-33. http:// dx.doi.org/10.1590/s1413-82712006000100004

Otto, M. W., Teachman, B. A., Cohen, L. S., Soares, C. N., Vitonis, A. F., \& Harlow, B. L. (2007). Dysfunctional attitudes and episodes of major depression: Predictive validity and temporal stability in never-depressed, depressed, and recovered women. Journal of Abnormal Psychology, 116(3), 475-483. http://dx.doi. org/10.1037/0021-843X.116.3.475

Power, M. J., Katz, R., McGuffin, P., Duggan, C. F., Lam, D., \& Beck, A. T. (1994). The Dysfunctionnal Attitude Scale (DAS). A comparison of forms $A$ and $B$ and proposals for a new subscaled version. Journal of Research in Personality, 28(3), 263-276. http://dx.doi. org/10.1006/jrpe.1994.1019

Rogers, G. M., Park, J., Essex, M. J., Klein, M. H., Silva, S. G., Hoyle, R. H., ... March, J. S. (2013). The Dysfunctional Attitudes Scale: Psychometric Properties in Depressed Adolescents. Journal of Clinical Child and Adolescent Psychology, 38(6), 781-789. http://dx.doi. org/10.1080/15374410903259007

Teodoro, M. L. M., \& Ohno, P. M. (2017). Desenvolvimento do sistema de crenças. In C. B. Neufeld, E. M. O.
Falcone, \& B. Rangé (Orgs.), PROCOGNITIVA Programa de Atualização em Terapia Cognitivo-Comportamental: ciclo 4. (Vol. 1, pp.9-54). Porto Alegre: Artmed.

Teodoro, M. L. M., Andrade, A. A., \& Castro, H. A. M. (2013). Escala de Pensamentos Automáticos para Crianças e Adolescentes (EPA): adaptação e propriedades psicométricas. Psico-USF, 18(1), 89-98. http://dx.doi. org/10.1590/S1413-82712013000100010

Teodoro, M. L. M., Froeseler, M. V. G., Almeida, V. M., \& Ohno, P. M. (2015). Inventário da Tríade Cognitiva para Crianças e Adolescentes (ITC-CA): adaptação e propriedades psicométricas. Avaliação Psicológica, 14(1), 63-72. http://dx.doi.org/10.15689/ ap.2015.1401.07

Villano, L. A. B., \& Nanhay, A. L. G. (2011). Depressão: epidemiologia e abordagem em cuidados primários de saúde. Revista Hospital Universitário Pedro Ernesto, 10(2), 10-20. http://dx.doi.org/0.1590/S1415-790X20 12000200012

Weissman, A. N., \& Beck, A. T. (1978). Development and validation of the Dysfunctional Attitudes Scale: A preliminary investigation. Chicago: Association for the Advancement of Behavior Therapy.

Young, C. C., LaMontagne, L. L., Dietrich, M. S., \& Wells, N. (2012). Cognitive vulnerabilities, negative life events, and depressive symptoms in young adolescents. Archives of Psychiatric Nursing, 26(1), 9-20. http://dx.doi.org/10.1016/j.apnu.2011.04.008

Recebido: julho 28, 2016

Versão final: julho 3, 2017

Aprovado: agosto 7, 2017 